\title{
Properties of the Sample Autocorrelations of Nonlinear Transformations in Long Memory Stochastic Volatility Models
}

\author{
ANA PÉREZ \\ Universidad de Valladolid, Spain \\ ESTHER RUIZ \\ Universidad Carlos III de Madrid, Spain
}

\begin{abstract}
The autocorrelations of log-squared, squared, and absolute financial returns are often used to infer the dynamic properties of the underlying volatility. This article shows that, in the context of long-memory stochastic volatility models, these autocorrelations are smaller than the autocorrelations of the log volatility and so is the rate of decay for squared and absolute returns. Furthermore, the corresponding sample autocorrelations could have severe negative biases, making the identification of conditional heteroscedasticity and long memory a difficult task. Finally, we show that the power of some popular tests for homoscedasticity is larger when they are applied to absolute returns.
\end{abstract}

KEYWORDS: absolute transformation, Box-Ljung text, conditional heteroscedasticity, log-squared transformation, Peña Rodriguez test, squared observations.

It is by now rather popular to measure the volatility of financial returns by some nonlinear transformations as, for example, log-squared, squared, or absolute returns. Consequently the autocorrelations of these transformations have often been used to test for the presence of conditional heteroscedasticity and to model and estimate the volatility [see, e.g., Harvey and Streibel (1998), Bollerslev and Mikkelsen (1999), Bollerslev and Wright (2000, 2001), and Baillie and Chung (2001) among many others]. In these studies, it is often concluded that log-squared, squared, or absolute returns are highly persistent processes. Therefore the dynamic evolution of volatility could be best described by a long-memory process [see

Financial support from projects BEC2002 03720 by the Spanish government and IHP RNT 002 by the European Community is acknowledged. This research was started while Ana Pérez was visiting the Department of Statistics and Econometrics (Universidad Carlos III de Madrid). She thanks the department for its hospitality. We are also grateful to A. Maravall, M. Regúlez, J. Rodríguez, S. Velilla, participants at the Econometric Society Australian Meeting at Brisbane and Simposio de Análisis Económico at Salamanca, the coeditor Eric Renault, and two anonymous referees, for their helpful comments and suggestions. Any remaining errors are our own. Address correspondence to Esther Ruiz, Dpto. Estadistica y Econometria, Universidad Carlos III de Madrid, c/Madrid, 126, 28903 Getafe, or e mail: ortega@est econ.uc3m.es. 
Dacorogna et al. (1993), Ding, Granger, and Engle (1993), Andersen and Bollerslev (1997), and Lobato and Savin (1998)]. The most popular long-memory models for volatilities are the fractionally integrated GARCH (FIGARCH) models of Baillie, Bollerslev, and Mikkelsen (1996) and Bollerslev and Mikkelsen (1996) and the long-memory stochastic volatility (LMSV) model proposed independently by Breidt, Crato, and de Lima (1998) and Harvey (1998). Little is known about the correlation structure of squares and other nonlinear transformations in FIGARCH models. Therefore we consider it more appropriate to investigate the behavior of the sample autocorrelations in LMSV models, where the dynamic properties of the squared, absolute, and log-squared transformations are already known.

In the context of long-memory linear processes, it is well known that the sample autocorrelations are negatively biased [see Newbold and Agiakloglou (1993) and Hosking (1996)]. However, this problem can be worsened in LMSV models because, as we have mentioned before, the volatility is not directly observable and it is often measured by transformations of returns that are highly noisy. In this article we show that the autocorrelations of these transformations are well below the autocorrelations of the corresponding volatility process. It is possible to find empirically relevant LMSV models where the volatility has true autocorrelations clearly different from zero, while those of log-squared, squared, and absolute returns are hardly different from zero. If, on top of that, the sample autocorrelations of these transformations are negatively biased, it is obvious that the identification and modelization of the dynamic evolution of volatility based on these sample autocorrelations is going to be severely affected. The objective of this article is twofold. First, we compare the true autocorrelations of the three transformations considered with those of the underlying log volatility. Second, we analyze the statistical properties of the corresponding sample autocorrelations and evaluate their reliability as a tool for identification and modeling of longmemory conditional heteroscedastic series generated by LMSV models.

The outline of the article is as follows. In Section 1, the correlation structure of the stationary LMSV process is presented. In this section we compare the autocorrelations of the three transformations of returns considered with the autocorrelations of the underlying log volatility process. Section 2 analyzes the asymptotic and finite sample properties of the sample autocorrelations of log-squared observations, while Section 3 investigates the performance of the sample autocorrelations of squared and absolute returns. The results of the corresponding Monte Carlo experiments show that the sample autocorrelations are negatively biased, making the identification of conditional heteroscedasticity a very difficult task. Section 4 analyzes the consequences of these biases on two tests commonly used to detect conditional heteroscedasticity, in particular, the Box-Ljung and the Peña and Rodriguez (2002) statistics. ${ }^{1}$ We analyze the finite sample size and power of

\footnotetext{
${ }^{1}$ In the context of GARCH models, Bollerslev (1988) and He and Terasvirta (1999) show that the correlo gram of the squared returns exhibits severe downward bias, even for large sample sizes. On the other hand, Bollerslev and Mikkelsen (1996) suggest that the conventional asymptotic chi square distribution of the Box Ljung statistic works well in testing homoscedasticity in GARCH models with long memory.
} 
these tests when they are implemented to test for the joint uncorrelatedness of the three transformations considered. Section 5 illustrates the results with the empirical analysis of daily returns of the Spanish stock market index IBEX-35 and the Standard \& Poor's 500 (S\&P 500) stock market index. Finally, Section 6 summarizes the main results.

\section{STATISTICAL PROPERTIES OF LMSV PROCESSES}

Stochastic volatility (SV) models represent the volatility of returns as an unobservable process, $\sigma_{\mathrm{t}}$. The simplest SV model, originally proposed by Taylor (1986), specifies the logarithm of $\sigma_{\mathrm{t}}$ as a linear autoregressive process and it is known as the autoregressive SV (ARSV) model. The long-memory property has been incorporated into SV models by Harvey (1998) and Breidt, Crato, and deLima (1998), who propose LMSV models where the log volatility follows an $\operatorname{ARFIMA}(p, d, q)$ process. In particular, when $p=1$ and $q=0$, the model for the series of returns, $y_{t}$, is given by

$$
\begin{aligned}
& y_{t}=\sigma_{*} \exp \left(h_{t} / 2\right) \varepsilon_{t} \\
& (1-\phi L)(1-L)^{d} h_{t}=\eta_{t},
\end{aligned}
$$

where $h_{t}=\log \left(\sigma_{\mathrm{t}}^{2}\right)$ is a scale factor that removes the need to include a constant term in Equation (1B), and $\phi$ and $d$ are the autoregressive and fractional parameters, respectively. The disturbances $\varepsilon_{t}$ and $\eta_{t}$ are mutually independent Gaussian white noise processes with zero mean and variances one and $\sigma_{\eta}^{2}$, respectively. Although the assumption of Gaussianity for $\eta_{t}$ can seem ad hoc, Andersen et al. (2001a, 2001b) show that the distribution of the log volatility can be well approximated by a normal distribution. On the other hand, the assumption of gaussianity for $\varepsilon_{t}$ in $\mathrm{SV}$ models is not as restrictive as it is in GARCH processes [see, Carnero, Peña, and Ruiz (2004)]. Finally, note that Model (1) is stationary if $|\phi|<1$ and $d<0.5$ and nests the short memory ARSV model when $d=0$.

With respect to the statistical properties of SV models, Ghysels, Harvey, and Renault (1996) show that the series $y_{t}$ is a martingale difference, but it is not an independent sequence. In fact, the autocorrelation function (ACF) of $\left|y_{t}\right|^{c}$ is given by

$$
\rho_{c}(k)=\frac{\exp \left(\left(c^{2} / 4\right) \sigma_{h}^{2} \rho_{h}(k)\right)-1}{\kappa_{c} \exp \left(\left(c^{2} / 4\right) \sigma_{h}^{2}\right)-1}, \quad \text { for } k \geq 1,
$$

where $\sigma_{h}^{2}$ and $\rho_{h}(k)$ are the variance and the ACF of the underlying log volatility, $h_{t}$ and $\kappa_{c}$ is a constant defined as

$$
\kappa_{c}=E\left(\left|\varepsilon_{t}\right|^{2 c}\right) /\left[E\left(\left|\varepsilon_{t}\right|^{c}\right)\right]^{2}=\Gamma\left(c+\frac{1}{2}\right) \Gamma\left(\frac{1}{2}\right) /\left[\Gamma\left(\frac{c}{2}+\frac{1}{2}\right)\right]^{2},
$$

where $\Gamma(\bullet)$ is the gamma function. For the cases of main interest, $c=1$ and $c=2$, this constant takes the values $\kappa_{1}=\pi / 2$ and $\kappa_{2}=3$, respectively. 
The variance and $\operatorname{ACF}$ of $\operatorname{ARFIMA}(p, d, q)$ processes have been derived by Hosking (1981). In particular, if $h_{t}$ is defined as in Equation (1B), its variance is given by

$$
\sigma_{h}^{2}=\sigma_{\eta}^{2} \frac{\Gamma(1-2 d)}{[\Gamma(1-d)]^{2}} \frac{F(1,1+d ; 1-d ; \phi)}{(1+\phi)},
$$

where $\mathrm{F}(\bullet, \bullet ; \bullet ; \bullet)$ is the hypergeometric function. Notice that when $\phi=0$, $F(1,1+d ; 1-d ; 0)=1$, and Equation (3) becomes the variance of an $\operatorname{ARFIMA}(0, d, 0)$ process, $\sigma_{h}^{2}=\sigma_{\eta}^{2} \Gamma(1-2 d) /[\Gamma(1-d)]^{2}$, as given by Harvey (1998). On the other hand, when $d=0, F(1,1 ; 1 ; \phi)=(1-\phi)^{-1}$, and Equation (3) becomes the variance of an AR(1) process, $\sigma_{h}^{2}=\sigma_{\eta}^{2} /\left(1-\phi^{2}\right)$, as in Harvey, Ruiz, and Shephard (1994). The $\mathrm{ACF}$ of $h_{t}$ is given by the following expression:

$$
\rho_{h}(k)=\left(\prod_{i}^{k} \frac{d+i}{1-d+i}\right) \frac{F(1, d+k ; 1-d+k ; \phi)+F(1, d-k ; 1-d-k ; \phi)-1}{(1-\phi) F(1,1+d ; 1-d ; \phi)}
$$

As $k \rightarrow \infty$, these autocorrelations behave like $\rho_{h}(k) \sim A k^{2 d-1}$, where $A$ is a factor of proportionality that depends on $d$ and $\phi$. Therefore the dependence between observations a long time span apart decays at a very slow hyperbolic rate. Finally, it is possible to show that when $d=0$, Equation (4) becomes the ACF of an AR(1) process, $\rho_{h}(k)=\phi^{k}$, and when $\phi=0$ it becomes the ACF of the ARFIMA $(0, d, 0)$ process, $\rho_{h}(k)=(d(d+1) \cdots(d+k-1)) /((1-d)(2-d) \cdots(k-d))$.

Andersen and Bollerslev (1997) and Robinson (2001) show that the autocovariances of squared and absolute returns decay at the same rate as the autocovariances of $h_{t}$ for large lags. This argument is often used to justify the use of these transformations to identify and model the long memory of volatility. However, the rate of decay of the autocorrelations of $\left|y_{t}\right|$ or $y_{t}^{2}$ and those of $h_{t}$ could be rather different for low lags. As an illustration, Figure 1(a) plots the rates of decay, defined as $\rho(k) / \rho(k-1)$, of the autocorrelations of $\left|y_{t}\right|$ or $y_{t}^{2}$ together with the rate of $h_{t}$ in an LMSV model with parameters $\left\{\phi=0.88, d=0.3, \sigma_{\eta}^{2}=0.026\right\}$ for different lags. This figure shows that, up to approximately lag 50 , the rate of decay of the autocorrelations of squares is clearly smaller than the rate of decay of the ACF of the log volatility. Therefore the autocorrelations of squares decay toward zero quicker than the autocorrelations of the log volatility. The rates of decay of the autocorrelations of both series are the same for large lags. The same behavior can be observed when comparing the rates of decay of the autocorrelations of absolute returns and log volatility autocorrelations, although in this case, they are closer than when comparing squared returns and log volatilities.

Another important difference between the ACF of $\left|y_{t}\right|$ or $y_{t}^{2}$ and the ACF of $h_{t}$ is the magnitude of the autocorrelations themselves, which are clearly smaller for $\left|y_{t}\right|$ and $y_{t}^{2}$ than for the log volatility process. This fact shows up in Figure 1(b), which displays the ACF of $h_{t}$ together with the ACF of $\left|y_{t}\right|$ and $y_{t}^{2}$ for the same model as before. In this case, the ACF of squared and absolute returns is nearly five times smaller than the ACF of the log volatilities. 
(a) Rate of convergence of the autocorrelations

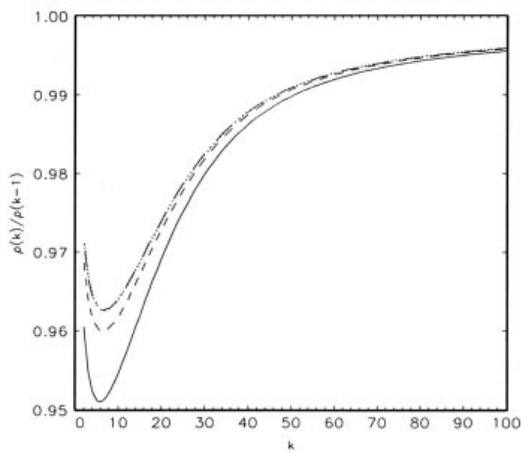

(b) Autocorrelation function

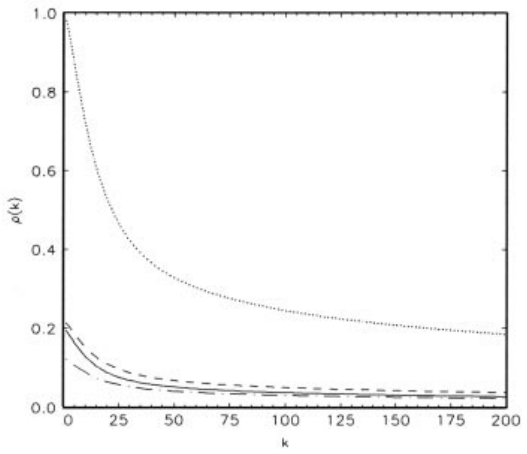

Figure 1 Comparison between rates of decay and magnitudes of autocorrelations of log squared ( . ), squared ( ), and absolute ( ) returns with respect to those of the underlying volatility $(\cdots)$ in an LMSV model with parameters $\left\{\begin{array}{llll}\phi & 0.88, d & 0.3, \sigma_{\eta}^{2} & 0.026\end{array}\right\}$.

(a) Autocorrelation function of $Y_{t}^{2}$

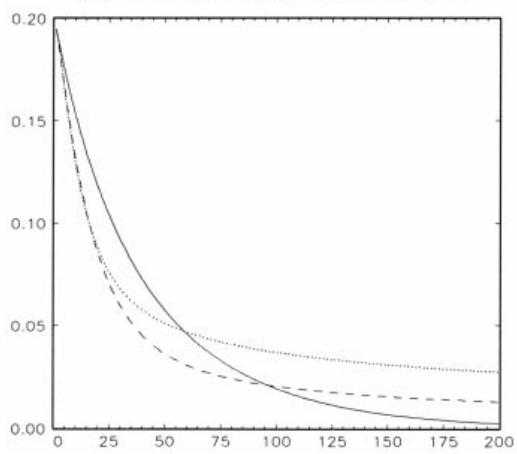

(b) Autocorrelation function of $\log \left(Y_{t}^{2}\right)$

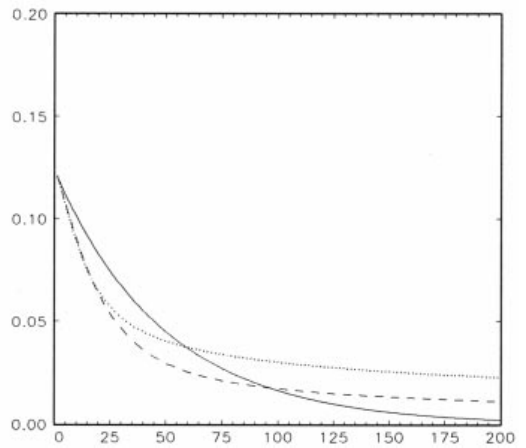

Figure 2 Autocorrelation function of $y_{t}^{2}$ and $\log \left(y_{t}^{2}\right)$ in LMSV processes with parameters $\{\phi$

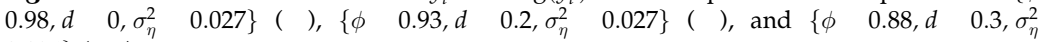
$0.026\}(\ldots)$.

It is also remarkable that the behavior of the ACF of short-memory and long-memory SV models can be rather similar in some cases. Figure 2(a) plots the ACF of $y_{t}^{2}$ for three LMSV models with parameters $\left\{\phi=0.98, d=0, \sigma_{\eta}^{2}=\right.$ $0.027\}$, and $\left\{\phi=0.93, d=0.2, \sigma_{\eta}^{2}=0.027\right\}$, and $\left\{\phi=0.88, d=0.3, \sigma_{\eta}^{2}=0.026\right\}$, respectively. These models are selected so that their coefficient of variation, defined as $\left\{\operatorname{var}\left(\sigma_{t}^{2}\right) /\left[E\left(\sigma_{t}^{2}\right)\right]^{2}\right\}$, is approximately one and the first-order autocorrelation of $y_{t}^{2}$ is 0.19 in all of them. Notice that, in practice, the rates of decay of the short-memory ARSV model and the LMSV model with small $d$ could be difficult to distinguish in the first lags. Indeed, the main differences only arise after the 
autocorrelation of approximately order 80 . Furthermore, observe that the autocorrelations up to order 20 of the two long-memory models displayed in Figure 2(a) are nearly indistinguishable. In these cases, the knowledge of the behavior in the long run will be essential. The same conclusions would be drawn if the ACF of absolute returns was used.

The dynamic dependence of the series of returns also appears in the logarithms of squares by considering the following linear representation of the LMSV process:

$$
\log \left(y_{t}^{2}\right)=\mu+h_{t}+\xi_{t}
$$

where $\mu=\log \left(\sigma_{*}^{2}\right)+E\left[\log \left(\varepsilon_{t}^{2}\right)\right], h_{t}$ is defined as in Equation (1B), and $\xi_{t}=\log \left(\varepsilon_{t}^{2}\right)-E\left[\log \left(\varepsilon_{t}^{2}\right)\right]$ is a non-Gaussian, zero mean, white noise process with variance $\sigma_{\xi}^{2}$ and independent of $h_{t}$. Consequently the variance of $\log \left(y_{t}^{2}\right)$ is $\gamma(0)=\sigma_{h}^{2}+\sigma_{\xi}^{2}$, its autocovariance function coincides with the autocovariance function of $h_{t}$, and its ACF is given by

$$
\rho(k)=\rho_{h}(k)\left\{1+\frac{\sigma_{\xi}^{2}}{\sigma_{h}^{2}}\right\}^{1}, \quad \text { for } k \geq 1 .
$$

Equation (6) states that the ACF of log-squared returns is proportional to the ACF of $h_{t}$, with the factor of proportionality being smaller than one. Therefore both ACF's decay at the same hyperbolic rate, but the ACF of $\log \left(y_{t}^{2}\right)$ takes smaller values. Figure 1(b) plots this ACF for the LMSV model with parameters $\left\{\phi=0.88, d=0.3, \sigma_{\eta}^{2}=0.026\right\}$. This figure shows that the ACF of $\log \left(y_{t}^{2}\right)$ takes even smaller values than the ACF of the other two transformations considered. Finally, Figure 2(b) plots the ACF of $\log \left(y_{t}^{2}\right)$ for the same three models considered in Figure 2(a). Again, this figure highlights the difficulty in distinguishing among different LMSV models using only the information contained in the ACF. As we will see in next section, this problem will be enlarged because of the negative bias of the sample ACF of $\log \left(y_{t}^{2}\right)$ in LMSV models.

\section{PROPERTIES OF THE SAMPLE AUTOCORRELATIONS OF LOG-SQUARED RETURNS}

In this section we analyze the asymptotic and finite sample properties of the sample autocorrelations of log-squared returns in stationary LMSV processes.

\subsection{Asymptotic Distribution}

The asymptotic distribution of the sample autocovariances of $\log \left(y_{t}^{2}\right)$ in LMSV models with $-0.5<d<0.25$ has been derived by Wright (1999). In particular, he shows that $\sqrt{ } T\{c(0)-\gamma(0), c(1)-\gamma(1), \ldots, c(k)-\gamma(k)\}$, where $c(k)$ and $\gamma(k)$ denote the sample and population autocovariance of order $k$, respectively, has 
an asymptotic multivariate normal distribution with zero mean and covariance matrix $V=\left[v_{i j}\right]_{i, j} \quad 0,1, \ldots, k$, whose elements are given by

$$
\begin{aligned}
v_{i j}= & \sum_{u}^{\infty}\left[\gamma_{h}(u) \gamma_{h}(u+i-j)+\gamma_{h}(u) \gamma_{h}(u+i+j)\right]+2 \sigma_{\xi}^{2}\left[\gamma_{h}(i-j)+\gamma_{h}(i+j)\right] \\
& \left.+\sigma_{\xi}^{4} I_{\{i j\}}+\left(\sigma_{\xi}^{4}+\pi^{4}\right) I_{\{i} j 0\right\}
\end{aligned}
$$

where $\gamma_{h}(\bullet)$ is the autocovariance function of $\left.h_{t}, I_{\{i} j\right\}$ is an indicator function defined as $\left.I_{\{i} j\right\}=1$ if $\left.i=j, I_{\{i} \quad j\right\}=0$ if $i \neq j$, and $I_{\{i \quad j \quad 0\}}$ is another indicator function defined similarly. Based on this result and using the theory on the convergence of differentiable functions of asymptotically normal vectors [Serfling (1980, p. 122)], it can be shown that the asymptotic distribution of $\sqrt{ } T\{r(1)-\rho(1), \ldots, r(k)-\rho(k)\}$, where $r(k)$ is the sample autocorrelation of order $k$, is multivariate normal with zero mean and covariance matrix $W=\left[w_{i j}\right]_{i, j} \quad 1, \ldots, k$, whose elements are given by

$$
\begin{aligned}
w_{i j}= & \sum_{u}^{\infty}\left[\rho(u) \rho(u+i-j)+\rho(u) \rho(u+i+j)+2 \rho(i) \rho(j) \rho^{2}(u)\right. \\
& -2 \rho(i) \rho(u) \rho(u+j)-2 \rho(j) \rho(u) \rho(u+i)]+\pi^{4} \frac{\rho(i) \rho(j)}{\gamma^{2}(0)}
\end{aligned}
$$

where $\gamma(0)$ and $\rho(k)$ are, respectively, the variance and the $k$ th autocorrelation of $\log \left(y_{t}^{2}\right)$.

The extra term that appears in Equation (7), if compared with the usual expression of the asymptotic covariances of $r(k)$ given by Anderson and Walker (1964) for linear models with i.i.d. disturbances, accounts for the non-Gaussianity of the added noise $\xi_{t}$ in Equation (5). In this case, Breidt and Davis (1992) show that the innovations of the linear representation of the series $\log \left(y_{t}^{2}\right)$ are uncorrelated but not independent. Therefore the conditions in Anderson and Walker (1964) do not hold and the classical theory for linear processes does not apply.

An alternative expression of the asymptotic variance of $r(k)$, which will be more useful in practice, can be obtained by taking $i=j=k$ in Equation (7) and rearranging terms as follows:

$$
\begin{aligned}
\operatorname{var}(r(k)) \sim & \frac{1}{T}\left\{1+2 \rho(2 k)+2 \rho^{2}(k)+2\left[1+2 \rho^{2}(k)\right] \sum_{u 1}^{\infty} \rho^{2}(u)\right. \\
& -4 \rho(k) \sum_{u=0}^{k} \rho(u) \rho(k-u)-8 \rho(k) \sum_{u 1}^{\infty} \rho(u) \rho(k+u) \\
& \left.+2 \sum_{u 1}^{\infty} \rho(u) \rho(u+2 k)+\sum_{u 1}^{2 k} \rho(u) \rho(2 k-u)+\pi^{4} \frac{\rho^{2}(k)}{\gamma^{2}(0)}\right\}
\end{aligned}
$$

In practice, the true values of $\rho(k)$ are unknown and the variance of $r(k)$ can be estimated from the data replacing $\rho(k)$ in Equation (8) by the corresponding 
sample autocorrelation, $r(k)$, and truncating the infinite summations at a sufficiently large lag. ${ }^{2}$

Finally, notice that the asymptotic distribution of $r(k)$ in stationary LMSV processes is only known when $d<0.25$. Hosking (1996) provides the asymptotic theory for the sample autocorrelations of a general class of long-memory linear processes with i.i.d. disturbances, that includes $\operatorname{ARFIMA}(p, d, q)$ processes with $-0.5<d<0.5$. However, as previously mentioned, the innovations of the linear representation of the series $\log \left(y_{t}^{2}\right)$ are uncorrelated but not independent, and therefore the conditions in Hosking (1996) do not hold and his results can not be directly applied to the LMSV process with $0.25 \leq d<0.5$. Further investigation is needed on this topic.

\subsection{Monte Carlo Results for the Sample Autocorrelations of $\log \left(y_{t}^{2}\right)$}

To assess the finite sample properties of the sample autocorrelations of logsquared observations generated by stationary LMSV models, we carry out Monte Carlo experiments with the following parameter specifications: $\phi=\{0$, $0.8,0.9,0.98\}, d=\{0,0.2,0.45\}$, and $\sigma_{\eta}^{2}=\{0.1,0.05,0.01\}$. These parameter values have been chosen so that they include empirically plausible models. In all cases, we assume that $\varepsilon_{t}$ is $\operatorname{NID}(0,1)$ and $\sigma_{*}=1$. Three sample sizes are considered, $T=512,1024$, and 4096. For each parameter set and sample size, 5000 independent replicates are generated, and for each replicate, the sample autocorrelations of $\log \left(y_{t}^{2}\right)$ up to order 50, are calculated.

All the simulations have been carried out using GAUSS version 3.2. The Gaussian noise, $\varepsilon_{t}$, and the AR(1) process in the ARSV model are generated with commands RNDNS and RECSERAR, respectively. In the models with long memory, the ARFIMA process is generated using the Davies and Harte (1987) algorithm. This algorithm requires the first $T$ autocovariances of the ARFIMA process in order to generate a series of length $T$. These autocovariances are calculated using the formulae in Chung (1994).

Table 1 reports the finite sample biases of the sample autocorrelations together with their empirical and asymptotic standard deviations (in parenthesis) for some selected models and lags $k=1,10$, and 50 . The asymptotic variance of $r(k)$ is calculated using Equation (8) and truncating the infinite summations at lag $M=20,000$. In LMSV models with $0.25 \leq d<0.5$, there is no guarantee that this formula applies, so the values of the asymptotic variance of $r(k)$ displayed for such models should be taken with much caution. Table 1 also displays the values of the $\mathrm{ACF}$ of the series $\log \left(y_{t}^{2}\right)$ calculated with Equation (6).

${ }^{2}$ For the ARSV process, the summations in Equation (8) can be worked out and the asymptotic variance of $r(k)$ can be written as a function of the parameters of the model as follows:

$$
\begin{aligned}
& \operatorname{var}(r(k)) \sim \frac{1}{T}\left\{1+\frac{2\left[1+2 \rho^{2}(k)\right]}{\gamma^{2}(0)} \frac{\phi^{2}}{1} \phi^{2} \sigma_{h}^{4}+\frac{\rho(2 k)}{\gamma(0)}\left[2 \sigma_{\xi}^{2}+\sigma_{h}^{2}\left(\frac{1+\phi^{2}}{1} \phi^{2}+2 k\right)\right]\right. \\
& \left.\frac{4 \rho^{2}(k)}{\gamma(0)}\left[2 \sigma_{\xi}^{2}+\sigma_{h}^{2}\left(\frac{1+\phi^{2}}{1 \quad \phi^{2}}+k\right)\right]+\rho^{2}(k)\left[2+\frac{\pi^{4}}{\gamma^{2}(0)}\right]\right\} \text {. }
\end{aligned}
$$


Table 1 Monte Carlo finite sample biases and standard deviations of sample autocorrelations of $\log \left(y_{t}^{2}\right)$ in LMSV models, together with their ACF values, for $k=1,10$, and 50 .

\begin{tabular}{|c|c|c|c|c|c|c|c|c|}
\hline \multirow[b]{2}{*}{$\phi, d, \sigma_{\eta}^{2}$} & \multirow[b]{2}{*}{ Lag } & \multirow[b]{2}{*}{$\mathrm{ACF}$} & \multicolumn{2}{|c|}{$T \quad 512$} & \multicolumn{2}{|c|}{ T 1024} & \multicolumn{2}{|c|}{ T 4096} \\
\hline & & & Bias & Std. dev. & Bias & Std. dev. & Bias & Std. dev. \\
\hline \multirow[t]{3}{*}{$0.98,0,0.05$} & 1 & 0.200 & 0.037 & $\begin{array}{c}0.075 \\
(0.086)\end{array}$ & 0.018 & $\begin{array}{c}0.056 \\
(0.061)\end{array}$ & 0.006 & $\begin{array}{c}0.030 \\
(0.031)\end{array}$ \\
\hline & 10 & 0.166 & 0.041 & $\begin{array}{c}0.074 \\
(0.087)\end{array}$ & 0.021 & $\begin{array}{c}0.056 \\
(0.061)\end{array}$ & 0.006 & $\begin{array}{c}0.030 \\
(0.031)\end{array}$ \\
\hline & 50 & 0.074 & 0.045 & $\begin{array}{c}0.060 \\
(0.082)\end{array}$ & 0.024 & $\begin{array}{c}0.050 \\
(0.058)\end{array}$ & 0.007 & $\begin{array}{c}0.028 \\
(0.029)\end{array}$ \\
\hline \multirow[t]{3}{*}{$0.9,0.2,0.01$} & 1 & 0.031 & 0.007 & $\begin{array}{c}0.045 \\
(0.047)\end{array}$ & 0.004 & $\begin{array}{c}0.032 \\
(0.033)\end{array}$ & 0.002 & $\begin{array}{c}0.016 \\
(0.016)\end{array}$ \\
\hline & 10 & 0.019 & 0.008 & $\begin{array}{c}0.045 \\
(0.046)\end{array}$ & 0.004 & $\begin{array}{c}0.032 \\
(0.032)\end{array}$ & 0.002 & $\begin{array}{c}0.016 \\
(0.016)\end{array}$ \\
\hline & 50 & 0.006 & 0.007 & $\begin{array}{c}0.042 \\
(0.045)\end{array}$ & 0.004 & $\begin{array}{c}0.031 \\
(0.032)\end{array}$ & 0.001 & $\begin{array}{c}0.016 \\
(0.016)\end{array}$ \\
\hline \multirow[t]{3}{*}{$0.9,0.2,0.1$} & 1 & 0.238 & 0.033 & $\begin{array}{c}0.066 \\
(0.081)\end{array}$ & 0.021 & $\begin{array}{c}0.049 \\
(0.058)\end{array}$ & 0.010 & $\begin{array}{c}0.026 \\
(0.029)\end{array}$ \\
\hline & 10 & 0.151 & 0.039 & $\begin{array}{c}0.064 \\
(0.084)\end{array}$ & 0.024 & $\begin{array}{c}0.048 \\
(0.059)\end{array}$ & 0.010 & $\begin{array}{c}0.026 \\
(0.029)\end{array}$ \\
\hline & 50 & 0.043 & 0.040 & $\begin{array}{c}0.055 \\
(0.076)\end{array}$ & 0.026 & $\begin{array}{c}0.044 \\
(0.060)\end{array}$ & 0.011 & $\begin{array}{c}0.025 \\
(0.030)\end{array}$ \\
\hline \multirow[t]{3}{*}{$0.8,0.45,0.01$} & 1 & 0.122 & 0.083 & $\begin{array}{c}0.047 \\
(0.608)\end{array}$ & 0.075 & $\begin{array}{c}0.035 \\
(0.430)\end{array}$ & 0.065 & $\begin{array}{c}0.020 \\
(0.215)\end{array}$ \\
\hline & 10 & 0.108 & 0.085 & $\begin{array}{c}0.047 \\
(0.618)\end{array}$ & 0.077 & $\begin{array}{c}0.035 \\
(0.437)\end{array}$ & 0.066 & $\begin{array}{c}0.020 \\
(0.218)\end{array}$ \\
\hline & 50 & 0.090 & 0.086 & $\begin{array}{c}0.042 \\
(0.630)\end{array}$ & 0.078 & $\begin{array}{c}0.033 \\
(0.445)\end{array}$ & 0.067 & $\begin{array}{c}0.019 \\
(0.223)\end{array}$ \\
\hline \multirow[t]{3}{*}{$0.9,0.45,0.01$} & 1 & 0.340 & 0.225 & $\begin{array}{c}0.065 \\
(1.369)\end{array}$ & 0.204 & $\begin{array}{c}0.053 \\
(0.968)\end{array}$ & 0.171 & $\begin{array}{c}0.038 \\
(0.484)\end{array}$ \\
\hline & 10 & 0.321 & 0.234 & $\begin{array}{c}0.064 \\
(1.408)\end{array}$ & 0.211 & $\begin{array}{c}0.053 \\
(0.996)\end{array}$ & 0.176 & $\begin{array}{c}0.039 \\
(0.498)\end{array}$ \\
\hline & 50 & 0.271 & 0.251 & $\begin{array}{c}0.053 \\
(1.512)\end{array}$ & 0.228 & $\begin{array}{c}0.049 \\
(1.069)\end{array}$ & 0.189 & $\begin{array}{c}0.039 \\
(0.534)\end{array}$ \\
\hline \multirow[t]{3}{*}{$0,0.45,0.1$} & 1 & 0.056 & 0.037 & $\begin{array}{c}0.044 \\
(0.280)\end{array}$ & 0.033 & $\begin{array}{c}0.032 \\
(0.198)\end{array}$ & 0.029 & $\begin{array}{c}0.016 \\
(0.099)\end{array}$ \\
\hline & 10 & 0.045 & 0.038 & $\begin{array}{c}0.044 \\
(0.284)\end{array}$ & 0.034 & $\begin{array}{c}0.032 \\
(0.200)\end{array}$ & 0.029 & $\begin{array}{c}0.016 \\
(0.100)\end{array}$ \\
\hline & 50 & 0.038 & 0.038 & $\begin{array}{c}0.041 \\
(0.286)\end{array}$ & 0.034 & $\begin{array}{c}0.031 \\
(0.202)\end{array}$ & 0.029 & $\begin{array}{c}0.016 \\
(0.101)\end{array}$ \\
\hline
\end{tabular}

Figures in parentheses are the asymptotic standard deviations calculated with Equation (8). 
Series $\log \left(y_{t}^{2}\right)$ with $T=512$

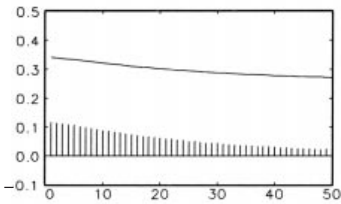

Series $y_{t}^{2}$ with $T=512$

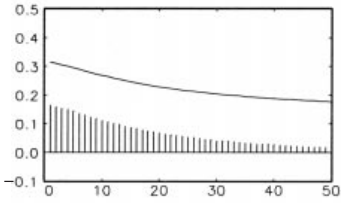

Series $|y$,$| with T=512$

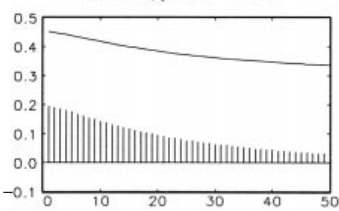

Series $\log \left(\mathrm{y}^{2}\right)$ with $T=1024$

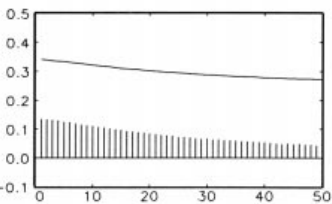

Series $y_{t}^{2}$ with $T=1024$

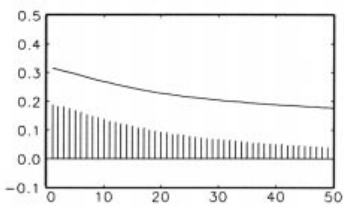

Series $\left|y_{1}\right|$ with $T=1024$

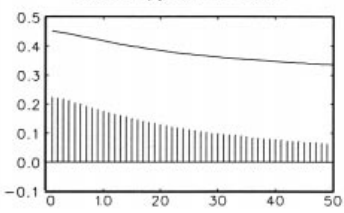

Series $\log \left(y_{t}^{2}\right)$ with $T=4096$

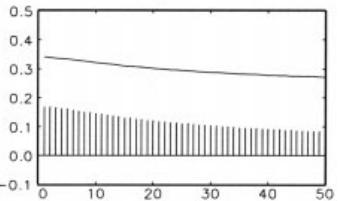

Series $y_{t}^{2}$ with $T=4096$

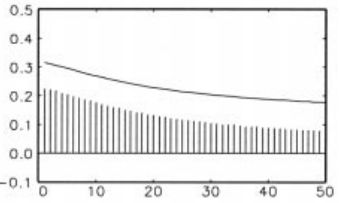

Series $|y$,$| with T=4096$

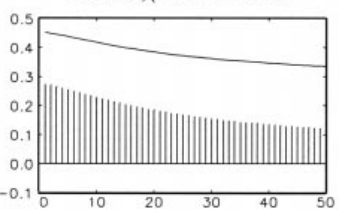

Figure 3 Mean correlogram (vertical bars) and autocorrelation function (solid line) of $\log \left(y_{t}^{2}\right), y_{t}^{2}$, and $\left|y_{t}\right|$ in an LMSV process with parameters $\left\{\begin{array}{llll}\phi & 0.9, d & 0.45, \sigma_{\eta}^{2} & 0.01\end{array}\right\}$ and sample sizes $T \quad\{512,1024,4096\}$.

As expected, the bias of $r(k)$ is always negative and depends on the parameter values. In the short-memory or less persistent models, when $d=0$ or $d=0.2$, the biases are negligible for moderate sample sizes. However, in the more persistent long-memory models, the biases can be rather important even for large sample sizes. For example, when $T=4096$, the relative bias of $r(1)$ in any of the models with $d=0.45$ is approximately $50 \%$. Furthermore, the relative bias increases with the lag. For instance, the relative biases of $r(1), r(10)$, and $r(50)$ in the LMSV model with parameters $\left\{\phi=0.8, d=0.45, \sigma_{\eta}^{2}=0.01\right\}$ and $T=1024$ are $61.5 \%, 71.3 \%$, and $86.7 \%$, respectively. In conclusion, the magnitude of the bias is such that using the correlogram of the log-squared observations as a tool for identifying LMSV models could be misleading. Finally, notice that even though the parameter specification $\left\{\phi=0.9, d=0.45, \sigma_{\eta}^{2}=0.01\right\}$ might seem too extreme, the effect of the bias makes the values of its mean sample autocorrelations very similar to those observed in real-time series of financial returns; see the top panels of Figure 3 which display the mean correlogram across replications, for $k=1, \ldots, 50$, together with the corresponding ACF of $\log \left(y_{t}^{2}\right)$ for this model and the three sample sizes considered.

With respect to the precision, the results in Table 1 show that the variance of $r(k)$ increases with $\phi, d$, and $\sigma_{\eta}^{2}$. Therefore the more persistent or the greater the 
variance of volatility, the less reliable is the correlogram as an estimator of the true ACF. Notice also that the usual asymptotic value $1 / \sqrt{ } T$ always underestimates the standard deviation of the sample autocorrelations of $\log \left(y_{t}^{2}\right)$. On the other hand, it seems that, in general, Equation (8) for the asymptotic variance of $r(k)$ provides an adequate approximation to the finite sample variance of $r(k)$ in ARSV and LMSV models with $0<d<0.25$. However, it clearly overestimates the empirical variance of the sample autocorrelations of $\log \left(y_{t}^{2}\right)$ when $0.25 \leq d<0.5$. For these parameter values, we have also computed the asymptotic variance using the formulae provided by Hosking (1996) for the sample autocorrelations in linear models with long memory, and we have found that they do not work in this case. This stresses the need for further investigation on the asymptotic distribution of $r(k)$ when $d \geq 0.25$.

Given that the bias is a serious problem, another interesting question to be investigated will be how to calculate unbiased sample autocorrelations of logsquared observations for LMSV models. Hosking (1996) derives an expression of this bias in linear long-memory processes assuming certain conditions on the coefficients and disturbances of the corresponding Wold representation of the process. However, this representation is not yet available for the reduced form of the log-squared observations of LMSV models. Consequently it is not possible to apply the expressions derived by Hosking (1996) to the bias of $r(k)$ in these models. However, in the short-memory ARSV model, it can be shown that the corresponding reduced form satisfies the conditions used by Fuller (1996, chap. 6) to derive the bias of the sample autocorrelations in linear processes. In this case, the application of Fuller's results leads to the following approximate expression of the bias of $r(k)$ :

$$
\begin{aligned}
E(r(k))-\rho(k) \approx & -\frac{\rho(k)}{T}\left[-k+2+4 \sum_{j 1}^{T}\left(1-\frac{j}{T}\right) \rho^{2}(j)\right] \\
& -\frac{1-\rho(k)}{T}\left[1+2 \sum_{j 1}^{T}\left(1-\frac{j}{T}\right) \rho(j)\right]+\frac{\rho(k)}{T} \frac{\pi^{4}}{\gamma^{2}(0)} \\
& -\frac{2}{T^{2}}\left[2 \sum_{j 1}^{T} \sum^{k 1}(T-k-j) \rho(j) \rho(k+j)+(T-k) \sum_{j 1}^{k} \rho(j) \rho(k+j)\right],
\end{aligned}
$$

where $\rho(k)$ is the ACF of $\log \left(y_{t}^{2}\right)$ in the ARSV model, that is, $\rho(k)=\phi^{k}\left\{1+\sigma_{\xi}^{2}\left(1-\phi^{2}\right) / \sigma_{\eta}^{2}\right\}^{1}$. As an illustration, Figure 4 displays, for an ARSV model with parameters $\left\{\phi=0.98, \sigma_{\eta}^{2}=0.05\right\}$ and sample size $T=1024$, the asymptotic standard normal density together with the empirical distribution across the 5000 replications of $\sqrt{ } T[r(k)-\rho(k)],[r(k)-\rho(k)] /[V(T, k)]^{1 / 2}$ and $[r(k)-\rho(k)-\operatorname{bias}(r(k))] /[V(T, k)]^{1 / 2}$, for $k=1$ and 10 , where $V(T, k)$ and bias $(r(k))$ denote the asymptotic variance of $r(k)$ and the bias of $r(k)$ given in Equations (8) and (9), respectively, calculated with the true parameter values. This figure confirms that the usual asymptotic variance, $1 / T$, is completely inappropriate for the sample autocorrelations of the log-squared observations. It also shows that there is 

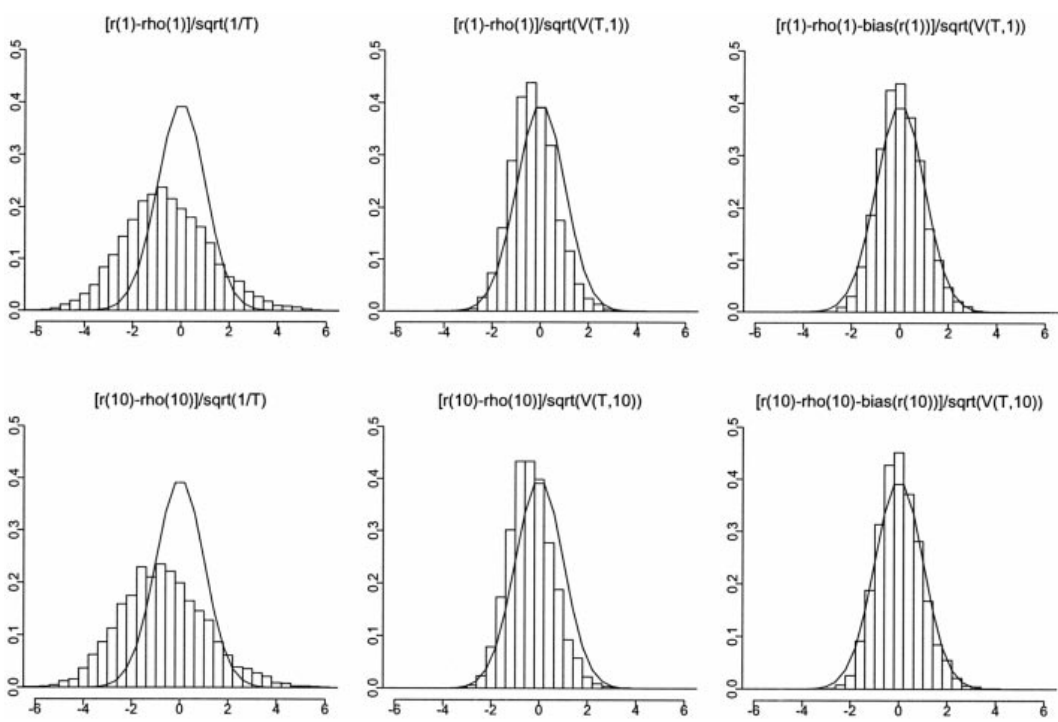

Figure 4 Standardized distributions of the sample autocorrelations of $\log \left(y_{t}^{2}\right)$ for lags $k \quad 1$ and 10 in an ARSV model with parameters $\left\{\begin{array}{lll}\phi & 0.98, \sigma_{\eta}^{2} & 0.05\end{array}\right\}$ and sample size $T$ 1024. From left to right the distributions are standardized with the usual $1 / T$ variance, with the asymptotic variance given in Equation (8), denoted by $V(T, k)$, and with the bias and asymptotic variance as given in Equations (9) and (8), respectively.

a remarkable improvement in the asymptotic approximation when the correction for bias is used. In practice, the true parameter values are unknown, and both the bias and variance of $r(k)$ must be estimated by replacing $\rho(k)$ in Equations (8) and (9) by its corresponding sample counterpart, $r(k)$. However, the distribution of $r(k)$ standardized with the estimated bias and variance does not provide good results in finite samples. In these cases, the bootstrap methodology proposed by Romano and Thombs (1996) would be a good alternative to approximate the empirical distribution of $r(k)$.

\section{FINITE SAMPLE PROPERTIES OF SAMPLE AUTOCORRELATIONS OF SQUARED AND ABSOLUTE RETURNS}

We now discuss the results for the squared and absolute series. In this case no asymptotic theory is available, ${ }^{3}$ so only finite sample properties are presented. Table 2 reports the empirical biases and standard deviations of the sample

\footnotetext{
${ }^{3}$ Davis and Mikosch (2000) provide some asymptotic results for the sample autocorrelations of absolute returns generated by a particular class of SV processes with infinite moments. However, their results do not apply to the LMSV considered in this article.
} 
Table 2 Monte Carlo finite sample biases and standard deviations of sample autocorrelations of $y_{t}^{2}$ and $\left|y_{t}\right|$ in LMSV models together with their ACF values, for $k=1,10$, and 50 .

\begin{tabular}{|c|c|c|c|c|c|c|c|c|c|}
\hline \multirow[b]{2}{*}{$\phi, d, \sigma_{\eta}^{2}$} & \multirow[b]{2}{*}{ Series } & \multirow[b]{2}{*}{ Lag } & \multirow[b]{2}{*}{$\mathrm{ACF}$} & \multicolumn{2}{|c|}{ T 512} & \multicolumn{2}{|c|}{ T 1024} & \multicolumn{2}{|c|}{ T 4096} \\
\hline & & & & Bias & Std. dev. & Bias & Std. dev. & Bias & Std. dev. \\
\hline \multirow[t]{6}{*}{$0.98,0,0.05$} & \multirow[t]{3}{*}{$y_{t}^{2}$} & 1 & 0.255 & 0.059 & 0.096 & 0.039 & 0.081 & 0.016 & 0.060 \\
\hline & & 10 & 0.188 & 0.059 & 0.081 & 0.037 & 0.070 & 0.014 & 0.050 \\
\hline & & 50 & 0.061 & 0.044 & 0.051 & 0.027 & 0.046 & 0.008 & 0.033 \\
\hline & \multirow[t]{3}{*}{$\left|y_{t}\right|$} & 1 & 0.314 & 0.065 & 0.092 & 0.037 & 0.071 & 0.011 & 0.043 \\
\hline & & 10 & 0.255 & 0.071 & 0.088 & 0.041 & 0.070 & 0.020 & 0.049 \\
\hline & & 50 & 0.106 & 0.070 & 0.068 & 0.041 & 0.060 & 0.018 & 0.047 \\
\hline \multirow[t]{6}{*}{$0.9,0.2,0.01$} & \multirow[t]{3}{*}{$y_{t}^{2}$} & 1 & 0.067 & 0.015 & 0.057 & 0.009 & 0.042 & 0.003 & 0.022 \\
\hline & & 10 & 0.041 & 0.014 & 0.050 & 0.009 & 0.038 & 0.003 & 0.020 \\
\hline & & 50 & 0.011 & 0.013 & 0.041 & 0.007 & 0.032 & 0.003 & 0.017 \\
\hline & \multirow{3}{*}{$\left|y_{t}\right|$} & 1 & 0.063 & 0.013 & 0.051 & 0.008 & 0.036 & 0.003 & 0.019 \\
\hline & & 10 & 0.040 & 0.013 & 0.048 & 0.008 & 0.035 & 0.003 & 0.018 \\
\hline & & 50 & 0.011 & 0.013 & 0.042 & 0.007 & 0.032 & 0.003 & 0.017 \\
\hline \multirow[t]{6}{*}{$0.9,0.2,0.1$} & \multirow[t]{3}{*}{$y_{t}^{2}$} & 1 & 0.268 & 0.041 & 0.108 & 0.029 & 0.092 & 0.011 & 0.070 \\
\hline & & 10 & 0.121 & 0.031 & 0.072 & 0.018 & 0.063 & 0.004 & 0.046 \\
\hline & & 50 & 0.024 & 0.026 & 0.044 & 0.015 & 0.038 & 0.005 & 0.026 \\
\hline & \multirow{3}{*}{$\left|y_{t}\right|$} & 1 & 0.353 & 0.053 & 0.085 & 0.034 & 0.066 & 0.013 & 0.038 \\
\hline & & 10 & 0.208 & 0.060 & 0.076 & 0.038 & 0.060 & 0.014 & 0.035 \\
\hline & & 50 & 0.055 & 0.053 & 0.060 & 0.035 & 0.051 & 0.014 & 0.033 \\
\hline \multirow{6}{*}{$0.8,0.45,0.01$} & \multirow[t]{3}{*}{$y_{t}^{2}$} & 1 & 0.197 & 0.119 & 0.065 & 0.106 & 0.050 & 0.086 & 0.032 \\
\hline & & 10 & 0.167 & 0.122 & 0.056 & 0.110 & 0.045 & 0.089 & 0.030 \\
\hline & & 50 & 0.132 & 0.126 & 0.044 & 0.113 & 0.038 & 0.093 & 0.026 \\
\hline & \multirow[t]{3}{*}{$\left|y_{t}\right|$} & 1 & 0.215 & 0.137 & 0.057 & 0.124 & 0.044 & 0.104 & 0.028 \\
\hline & & 10 & 0.188 & 0.142 & 0.053 & 0.129 & 0.042 & 0.108 & 0.027 \\
\hline & & 50 & 0.156 & 0.148 & 0.045 & 0.134 & 0.038 & 0.112 & 0.026 \\
\hline \multirow[t]{6}{*}{$0.9,0.45,0.01$} & \multirow[t]{3}{*}{$y_{t}^{2}$} & 1 & 0.314 & 0.150 & 0.089 & 0.126 & 0.074 & 0.090 & 0.054 \\
\hline & & 10 & 0.268 & 0.157 & 0.075 & 0.132 & 0.065 & 0.094 & 0.049 \\
\hline & & 50 & 0.177 & 0.161 & 0.051 & 0.138 & 0.048 & 0.101 & 0.039 \\
\hline & \multirow[t]{3}{*}{$\left|y_{t}\right|$} & 1 & 0.451 & 0.258 & 0.083 & 0.227 & 0.068 & 0.178 & 0.049 \\
\hline & & 10 & 0.418 & 0.275 & 0.078 & 0.242 & 0.067 & 0.189 & 0.050 \\
\hline & & 50 & 0.336 & 0.307 & 0.063 & 0.273 & 0.060 & 0.215 & 0.051 \\
\hline \multirow[t]{6}{*}{$0,0.45,0.1$} & \multirow[t]{3}{*}{$y_{t}^{2}$} & 1 & 0.105 & 0.064 & 0.054 & 0.057 & 0.040 & 0.048 & 0.023 \\
\hline & & 10 & 0.081 & 0.065 & 0.048 & 0.059 & 0.036 & 0.049 & 0.021 \\
\hline & & 50 & 0.068 & 0.066 & 0.042 & 0.059 & 0.033 & 0.050 & 0.019 \\
\hline & \multirow[t]{3}{*}{$\left|y_{t}\right|$} & 1 & 0.107 & 0.068 & 0.049 & 0.061 & 0.036 & 0.052 & 0.020 \\
\hline & & 10 & 0.085 & 0.069 & 0.047 & 0.062 & 0.034 & 0.053 & 0.019 \\
\hline & & 50 & 0.072 & 0.070 & 0.042 & 0.063 & 0.033 & 0.053 & 0.018 \\
\hline
\end{tabular}


autocorrelations of the squared and absolute returns for lags $k=1,10$, and 50 and for the same specifications as in Table 1. It also displays the values of the ACF of $y_{t}^{2}$ and $\left|y_{t}\right|$ given in Equation (2).

In general, the results reported in Table 2 are similar to the ones obtained for $\log$-squared returns. The relative biases of the sample autocorrelations of $y_{t}^{2}$ are comparable with the ones obtained for $\log \left(y_{t}^{2}\right)$, although they are slightly larger in the short-memory model and smaller in the models with long memory. For example, for the LMSV model with parameters $\left\{\phi=0.8, d=0.45, \sigma_{\eta}^{2}=0.01\right\}$ and $T=1024$, the relative biases of the first-order autocorrelation of squared and log-squared returns are $53.8 \%$ and $61.5 \%$, respectively. On the other hand, the standard deviations of the low-order sample autocorrelations are always larger for squared than for log-squared returns. Therefore it is not clear whether it is more convenient to use $\log \left(y_{t}^{2}\right)$ or $\left(y_{t}^{2}\right)$ in order to identify conditional heteroscedasticity in the context of LMSV models.

With respect to the absolute returns, their sample autocorrelations are again downward biased and their biases are important and more severe the more persistent the volatility and the greater its variance. In general, the relative biases of the absolute and squared returns are similar. For example, in the model with $\left\{\phi=0.9, d=0.45, \sigma_{\eta}^{2}=0.01\right\}$ and $T=1024$, the relative bias of $r(1)$ for squared returns is $40.13 \%$ while for absolute returns it is $50.33 \%$. As in the squared series, in the ARSV model, the biases become negligible for large sample sizes. Also, in the LMSV model with $d=0.2$ and $\sigma_{\eta}^{2}=0.01$, the biases are negligible because the autocorrelations themselves are nearly zero and the series is close to a white noise process. However, this is not the case in the more persistent LMSV models, where the biases remain important, even for sample sizes as large as $T=4096$. The bottom panels of Figure 3 illustrate this point by showing the mean correlogram across replications and the true ACF of $\left|y_{t}\right|$ for the LMSV process with $\left\{\phi=0.9, d=0.45, \sigma_{\eta}^{2}=0.01\right\}$ and the three sample sizes considered.

Comparing the standard deviations of the low-order sample autocorrelations of absolute and squared returns, it can be observed that they are smaller for the former. However, the variance of $r(50)$ for absolute returns is similar or even larger than for the squared series. This is due to the fact that the variance of the sample autocorrelations of absolute returns remains nearly the same regardless of the lag considered, but it decreases rapidly with the lag for the squared transformation. As expected, in all cases, the variance of $r(k)$ decreases as the sample size increases, but the rate of convergence is smaller than $T$.

Finally, notice that for the empirically plausible models considered, the population autocorrelations are clearly larger for absolute returns than for the other two transformations. Therefore it seems that their sample autocorrelations are more reliable to identify the presence of heteroscedasticity in the context of LMSV models. Nevertheless, as we have already mentioned, in the more persistent long-memory cases $(d=0.45)$, the biases in the three transformations considered are always negative and quite important, and they remain nearly the same regardless of the order of the autocorrelation being estimated. Moreover, the magnitude of the bias hardly decreases as the sample size increases, as illustrated in Figure 3. 
As a consequence, the sample autocorrelation of these three transformations are not reliable estimators of their population counterparts and should not be used as an identification tool in LMSV models with high persistence in the volatility.

\section{FINITE SAMPLE PROPERTIES OF HOMOSCEDASTICITY TESTS}

A very popular test for uncorrelatedness is the one based on the Box-Ljung statistic given by

$$
Q(K)=T(T+2) \sum_{j 1}^{K} \frac{r^{2}(j)}{T-j^{\prime}}
$$

where $r(j)$ is the $j$ th sample autocorrelation of the series to be tested for uncorrelatedness. Under the null hypothesis of the first $K$ autocorrelations being jointly equal to zero, McLeod and $\mathrm{Li}$ (1983) show that the asymptotic distribution of $Q(K)$, when applied to squared observations is approximately a chi-square distribution with $K$ degrees of freedom if the eighth-order moment of $y_{t}$ exists. This result is based on Theorem 14 of Hannan (1970, p. 228) that requires the series to be tested for uncorrelatedness to be an independent sequence with finite fourth-order moment. Consequently, notice that the asymptotic distribution of $Q(K)$, when applied to absolute and log-squared returns, is also a chi-square distribution with $K$ degrees of freedom if the fourth-order moment of $\left|y_{t}\right|$ and $\log \left(y_{t}^{2}\right)$, respectively, exists.

Alternatively, Peña and Rodriguez (2002) have proposed a new portmanteau test based on the Kth root of the determinant of the Kth autocorrelation matrix. The proposed statistic is given by

$$
D(K)=T\left[1-\left|R_{K}\right|^{1 / K}\right]
$$

where

$$
R_{K}=\left[\begin{array}{cccc}
1 & \tilde{r}(1) & \ldots & \tilde{r}(K) \\
\tilde{r}(1) & 1 & \ldots & \tilde{r}(K-1) \\
\ldots & \ldots & \ldots & \ldots \\
\tilde{r}(K) & \tilde{r}(K-1) & \ldots & 1
\end{array}\right]
$$

and $\tilde{r}(j)$ is the estimated standardized autocorrelation coefficient of order $j$ given by $\tilde{r}(j)=\sqrt{ }(T+2) /(T-j) r(j)$. When applied to squared observations, Peña and Rodriguez (2002) show that the asymptotic distribution of the statistic can be approximated by a gamma distribution, $\gamma(\alpha, \beta)$, with $\alpha=3 K(K+1) / 4(2 K+1)$ and $\beta=3 K / 2(2 K+1)$ for moderate values of $\alpha$. This result can also be applied to the absolute value and log-squared transformations if the corresponding fourthorder moments exist.

In this section we carry out Monte Carlo experiments to assess the finite sample properties of both the $Q(K)$ and $D(K)$ statistics when they are implemented as portmanteau tests for the joint significance of the autocorrelations of squared, 
Table 3 Empirical sizes of Box-Ljung $Q(K)$ and Peña-Rodriguez $D(K)$ tests for joint uncorrelatedness in nonlinear transformations of Gaussian, Student's $t_{5}$, and Student's $t_{9}$ independent white noise, with $K=10$ and 50 .

\begin{tabular}{|c|c|c|c|c|c|c|c|c|c|c|}
\hline \multirow[b]{2}{*}{ Test } & \multirow[b]{2}{*}{ Series } & \multicolumn{3}{|c|}{$T \quad 512$} & \multicolumn{3}{|c|}{ T 1024} & \multicolumn{3}{|c|}{ T 4096} \\
\hline & & Gaussian & $t_{5}$ & $t_{9}$ & Gaussian & $t_{5}$ & $t_{9}$ & Gaussian & $t_{5}$ & $t_{9}$ \\
\hline \multirow[t]{3}{*}{$Q(10)$} & $\log \left(y_{t}^{2}\right)$ & 0.047 & 0.051 & 0.052 & 0.047 & 0.058 & 0.051 & 0.052 & 0.049 & 0.060 \\
\hline & $y_{t}^{2}$ & 0.049 & 0.065 & 0.055 & 0.051 & 0.068 & 0.057 & 0.057 & 0.072 & 0.055 \\
\hline & $\left|y_{t}\right|$ & 0.050 & 0.049 & 0.049 & 0.050 & 0.048 & 0.046 & 0.053 & 0.047 & 0.049 \\
\hline \multirow[t]{3}{*}{$Q(50)$} & $\log \left(y_{t}^{2}\right)$ & 0.062 & 0.059 & 0.065 & 0.055 & 0.059 & 0.066 & 0.054 & 0.052 & 0.050 \\
\hline & $y_{t}^{2}$ & 0.061 & 0.065 & 0.060 & 0.052 & 0.082 & 0.060 & 0.054 & 0.099 & 0.063 \\
\hline & $\left|y_{t}\right|$ & 0.063 & 0.053 & 0.059 & 0.056 & 0.050 & 0.057 & 0.048 & 0.052 & 0.049 \\
\hline \multirow[t]{3}{*}{$D(10)$} & $\log \left(y_{t}^{2}\right)$ & 0.044 & 0.046 & 0.048 & 0.042 & 0.051 & 0.051 & 0.056 & 0.049 & 0.053 \\
\hline & $y_{t}^{2}$ & 0.046 & 0.064 & 0.055 & 0.050 & 0.063 & 0.055 & 0.056 & 0.067 & 0.055 \\
\hline & $\left|y_{t}\right|$ & 0.049 & 0.048 & 0.048 & 0.042 & 0.043 & 0.050 & 0.052 & 0.048 & 0.049 \\
\hline \multirow[t]{3}{*}{$D(50)$} & $\log \left(y_{t}^{2}\right)$ & 0.035 & 0.034 & 0.037 & 0.041 & 0.045 & 0.045 & 0.049 & 0.050 & 0.054 \\
\hline & $y_{t}^{2}$ & 0.038 & 0.056 & 0.047 & 0.044 & 0.072 & 0.054 & 0.053 & 0.094 & 0.049 \\
\hline & $\left|y_{t}\right|$ & 0.039 & 0.034 & 0.037 & 0.040 & 0.041 & 0.044 & 0.051 & 0.051 & 0.044 \\
\hline
\end{tabular}

Nominal size is $5 \%$.

log-squared, or absolute returns. ${ }^{4}$ In order to assess the size of both tests, we have generated homoscedastic serially independent series using three different distributions, a standard normal and two Student's $t$ distributions with $\nu=5$ and $\nu=9$ degrees of freedom, respectively, standardized to have unit variance. The Student's $t$ distributions have been chosen because it has often been observed that the marginal distribution of returns has heavy tails. Moreover, the degrees of freedom have been selected so that we have a distribution with a finite eighth-order moment $(\nu=9)$, as required by McLeod and Li (1983), and a distribution that does not satisfy this requirement $(\nu=5)$. On the other hand, the powers of the $Q(K)$ and $D(K)$ statistics have been analyzed, generating heteroscedastic series by the same LMSV models and sample sizes considered in Sections 2 and 3. All the results are based on 5000 replicates.

Table 3 reports the empirical rejection probabilities of the $Q(K)$ and $D(K)$ tests, for $K=10$ and 50, implemented with log-squared, squared, and absolute returns generated by the homoscedastic white noise processes previously described. In all cases, the nominal size is $\alpha=0.05$. This table shows that, in general, the empirical

\footnotetext{
${ }^{4}$ In practice, the series of returns may contain zeroes and/or values very close to zero, and the log squared transformation could break down. To overcome this problem, Fuller (1996) proposes an adjustment of such transformation given by $x_{t}^{*}=\log \left(y_{t}^{2}+\tau s^{2}\right) \quad \tau s^{2} /\left(y_{t}^{2}+\tau s^{2}\right)$, where $s^{2}$ is the sample variance of $y_{t}$ and $\tau$ is a subjectively chosen small constant that Fuller (1996) and Breidt and Carriquiry (1996) fix as $\tau=0.02$. The $Q(K)$ test is more powerful against long memory alternatives if applied to this transformation than to the log squared returns. However, given that it still provides lower power compared to the absolute transformation, we do not report the corresponding results, although they are available upon request.
} 

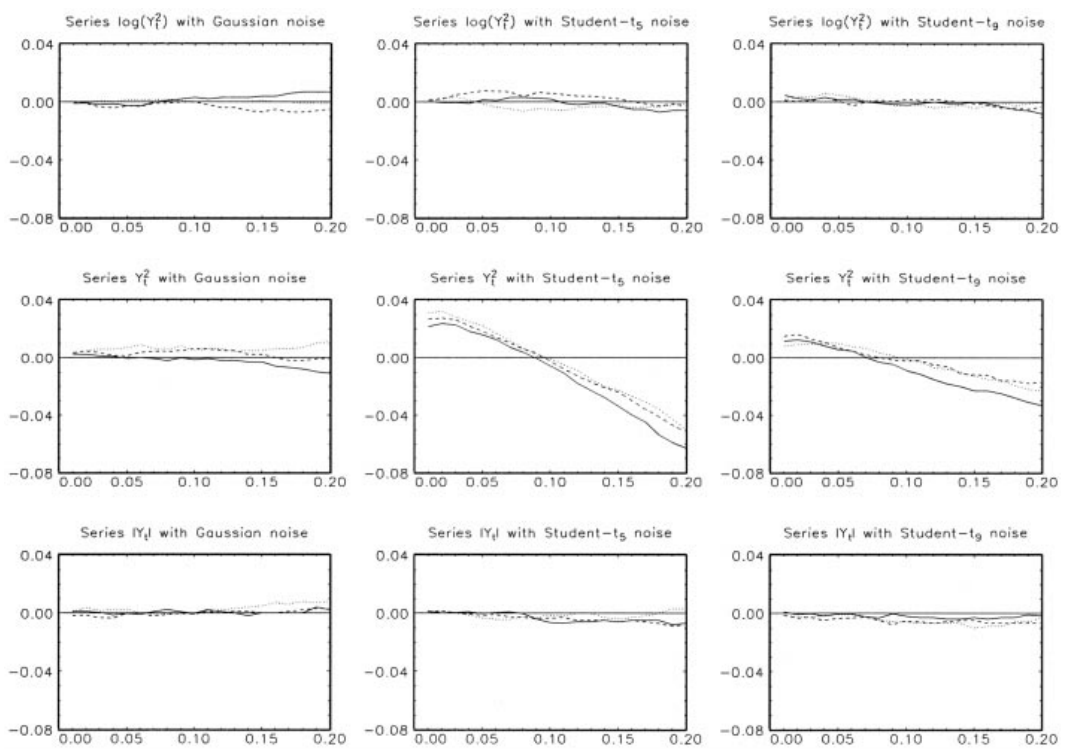

Figure 5 Differences between empirical and nominal rejection probabilities of the $Q(10)$ test for nonlinear transformations of Gaussian and Student's $t$ noises with $T$ 512( ), $T$ 1024 ( ), and T $4096(\cdots)$.

sizes of the $Q(10)$ and $D(10)$ statistics are close to the nominal size for all the transformations considered, except for squared returns generated by the Student's $t_{5}$ white noise, where the empirical sizes are always larger than the nominal size. Moreover, the larger the sample size, the larger the gap between them. This situation becomes more dramatic when the $Q(50)$ and $D(50)$ tests are used. In these cases, the empirical size nearly doubles the nominal when $T=1024$ or $T=4096$. Therefore, even if the sample size is very large, the null of conditional homoscedasticity may be rejected in the presence of homoscedastic series with heavy-tailed marginal distributions if squared returns are used. This problem is further illustrated in Figure 5, which plots the differences between the empirical and nominal sizes of the $Q(10)$ test for log-squared, squared, and absolute returns for values of the nominal rejection probabilities that span $\alpha=0.01$ to 0.2 and for the three sample sizes considered. This figure shows that when the series $y_{t}$ is a Gaussian noise, the $Q(10)$ test keeps the nominal size quite well, whatever sample size and transformation is used. However, when the series is generated by a Student's $t_{5}$ noise, there are important differences between the nominal and the empirical rejection probabilities for squared returns. The empirical size is smaller than the nominal when $\alpha>0.10$ and is larger than the nominal when $\alpha<0.10$ for any sample size. When the tests are applied to nonlinear transformations of a Student's $t_{9}$ noise, some discrepancies appear in the squares for the larger nominal sizes, but for the most commonly used values, the empirical size is close to the nominal. 
Table 4 Empirical powers of Box-Ljung $Q(K)$ and Peña-Rodriguez $D(K)$ tests for nonlinear transformations of LMSV processes, with $K=10$ and 50.

\begin{tabular}{|c|c|c|c|c|c|c|c|c|c|c|}
\hline \multirow[b]{2}{*}{$\phi, d, \sigma_{\eta}^{2}$} & \multirow[b]{2}{*}{ Test } & \multicolumn{3}{|c|}{ T 512} & \multicolumn{3}{|c|}{ T 1024} & \multicolumn{3}{|c|}{ T 4096} \\
\hline & & $\log \left(y_{t}^{2}\right)$ & $y_{t}^{2}$ & $\left|y_{t}\right|$ & $\log \left(y_{t}^{2}\right)$ & $y_{t}^{2}$ & $\left|y_{t}\right|$ & $\log \left(y_{t}^{2}\right)$ & $y_{t}^{2}$ & $\left|y_{t}\right|$ \\
\hline \multirow[t]{4}{*}{$0.98,0,0.05$} & $Q(10)$ & 0.968 & 0.994 & 0.999 & 1 & 1 & 1 & 1 & 1 & 1 \\
\hline & $Q(50)$ & 0.947 & 0.983 & 0.995 & 0.999 & 1 & 1 & 1 & 1 & 1 \\
\hline & $D(10)$ & 0.957 & 0.992 & 0.999 & 1 & 1 & 1 & 1 & 1 & 1 \\
\hline & $D(50)$ & 0.916 & 0.982 & 0.995 & 0.999 & 1 & 1 & 1 & 1 & 1 \\
\hline \multirow{4}{*}{$0.9,0.2,0.01$} & $Q(10)$ & 0.142 & 0.498 & 0.438 & 0.269 & 0.796 & 0.759 & 0.846 & 1 & 1 \\
\hline & $Q(50)$ & 0.117 & 0.384 & 0.337 & 0.210 & 0.690 & 0.633 & 0.760 & 0.999 & 0.999 \\
\hline & $D(10)$ & 0.112 & 0.455 & 0.388 & 0.234 & 0.768 & 0.715 & 0.822 & 1 & 1 \\
\hline & $D(50)$ & 0.074 & 0.376 & 0.277 & 0.173 & 0.703 & 0.606 & 0.782 & 1 & 1 \\
\hline \multirow[t]{4}{*}{$0.9,0.2,0.1$} & $Q(10)$ & 0.994 & 0.996 & 1 & 1 & 1 & 1 & 1 & 1 & 1 \\
\hline & $Q(50)$ & 0.982 & 0.973 & 1 & 1 & 0.997 & 1 & 1 & 1 & 1 \\
\hline & $D(10)$ & 0.942 & 0.997 & 1 & 1 & 1 & 1 & 1 & 1 & 1 \\
\hline & $D(50)$ & 0.976 & 0.977 & 1 & 1 & 0.998 & 1 & 1 & 1 & 1 \\
\hline \multirow[t]{4}{*}{$0.8,0.45,0.01$} & $Q(10)$ & 0.304 & 0.726 & 0.707 & 0.628 & 0.965 & 0.963 & 0.999 & 1 & 1 \\
\hline & $Q(50)$ & 0.267 & 0.644 & 0.620 & 0.576 & 0.941 & 0.935 & 0.999 & 1 & 1 \\
\hline & $D(10)$ & 0.254 & 0.697 & 0.662 & 0.566 & 0.954 & 0.950 & 0.999 & 1 & 1 \\
\hline & $D(50)$ & 0.175 & 0.621 & 0.551 & 0.499 & 0.939 & 0.925 & 0.999 & 1 & 1 \\
\hline \multirow[t]{4}{*}{$0.9,0.45,0.01$} & $Q(10)$ & 0.835 & 0.990 & 0.988 & 0.994 & 1 & 1 & 1 & 1 & 1 \\
\hline & $Q(50)$ & 0.781 & 0.972 & 0.972 & 0.990 & 1 & 1 & 1 & 1 & 1 \\
\hline & $D(10)$ & 0.860 & 0.984 & 0.990 & 0.995 & 1 & 1 & 1 & 1 & 1 \\
\hline & $D(50)$ & 0.772 & 0.971 & 0.977 & 0.993 & 1 & 1 & 1 & 1 & 1 \\
\hline \multirow[t]{4}{*}{$0,0.45,0.1$} & $Q(10)$ & 0.095 & 0.294 & 0.249 & 0.180 & 0.550 & 0.516 & 0.711 & 0.995 & 0.995 \\
\hline & $Q(50)$ & 0.094 & 0.259 & 0.224 & 0.178 & 0.521 & 0.479 & 0.740 & 0.994 & 0.993 \\
\hline & $D(10)$ & 0.077 & 0.281 & 0.230 & 0.160 & 0.535 & 0.492 & 0.688 & 0.994 & 0.993 \\
\hline & $D(50)$ & 0.051 & 0.236 & 0.162 & 0.130 & 0.508 & 0.427 & 0.700 & 0.993 & 0.993 \\
\hline
\end{tabular}

Nominal size is $5 \%$.

Next we analyze the finite sample power of both the $Q(K)$ and $D(K)$ tests against LMSV alternatives using the same designs as in Sections 2 and 3. The results for $K=10$ and 50 when the nominal size is $5 \%$ are reported in Table 4 . The main conclusions we can draw from this table are as follows. The powers of the Peña-Rodriguez statistic are similar or slightly lower than those of the BoxLjung statistic for all the designs, sample sizes, and transformations considered. This result agrees with the conclusions of Peña and Rodriguez (2002), who carry out a small Monte Carlo experiment using the squares of ARSV processes. On the other hand, the behavior of both statistics across the three transformations considered are similar, so we will focus on describing the results for the Box-Ljung statistic. First, it is important to observe that, regardless of the design, sample size, and number of lags considered, when the test for uncorrelatedness is carried out 

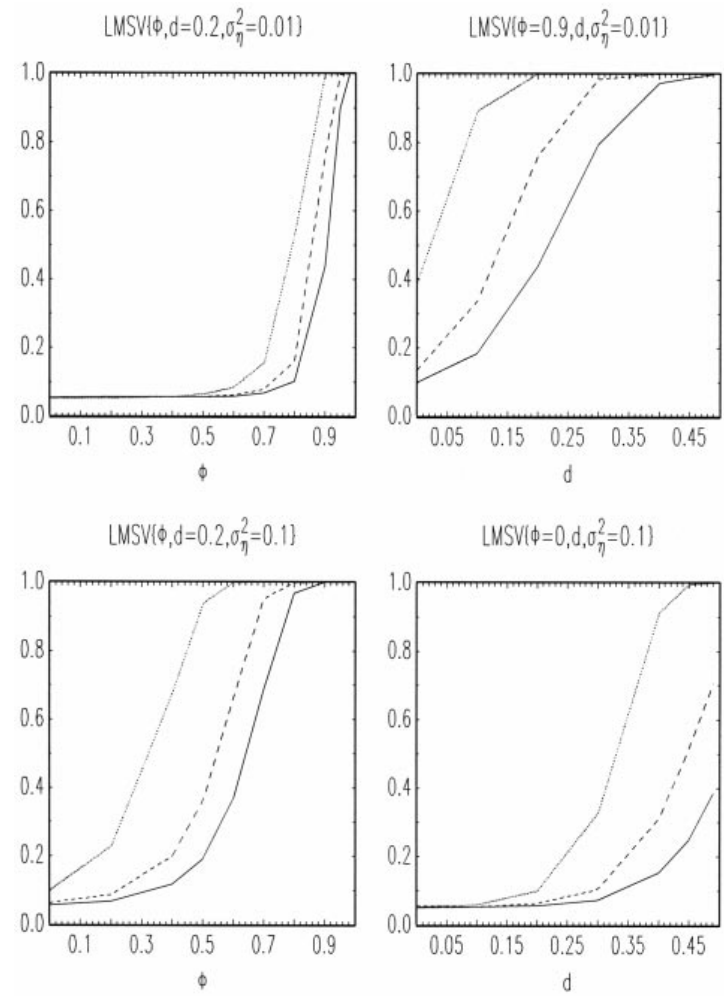

Figure 6 Empirical powers of the $Q(10)$ test for absolute returns in LMSV processes with $T 512(\mathrm{r}), \mathrm{T} 1024$ ( ), and $T 4096(\ldots)$ Nominal size is $5 \%$.

for log-squared returns, the powers are always smaller than for squared and absolute returns. Moreover, for these last two transformations, the powers are comparable. In any case, when the series $y_{t}$ is generated by the ARSV model with sample sizes as large as $T=1024$ or $T=4096$, it is clear that it does not matter which transformation is used to test for conditional homoscedasticity, because the empirical powers are essentially equal to one. A different situation arises in the LMSV process with $d=0.2$ and $\sigma_{\eta}^{2}=0.01$. In this case, the powers are low because this process stands for a situation very close to a homoscedastic white noise where the autocorrelations themselves are nearly zero. When the series is generated by the very persistent LMSV model with $\left\{\phi=0, d=0.45, \sigma_{\eta}^{2}=0.1\right\}$, the powers are also rather low because of the negative biases in the sample autocorrelations. The same features can be observed in the LMSV process with $\left\{\phi=0.8, d=0.45, \sigma_{\eta}^{2}=0.01\right\}$. For these alternatives, large sample sizes are needed to obtain satisfactory powers.

To illustrate the dependence of the power on the parameters of the model, Figure 6 displays the empirical powers of the $Q(10)$ test for absolute returns and 
the three sample sizes considered when the rejection probability is $5 \%$. The panels on the left plot the power as a function of the autoregressive parameter, $\phi$, when $d=0.2$ and $\sigma_{\eta}^{2}=0.01$ (top panel) and $\sigma_{\eta}^{2}=0.1$ (bottom panel). Unlike in the righthand-side panels, the power is represented as a function of the long-memory parameter, $d$, when the other parameters are fixed to $\left\{\phi=0.9, \sigma_{\eta}^{2}=0.01\right\}$ and $\left\{\phi=0, \sigma_{\eta}^{2}=0.1\right\}$ on the top and bottom panels, respectively. Notice that in LMSV processes, the series $y_{t}$ becomes a white noise only when both parameters, $\phi$ and $d$, equal zero. Therefore, if only one of these parameters is zero, the model is not under the null of homoscedasticity and consequently the empirical power function does not start at the nominal 5\%. The plots in Figure 6 show that the heteroscedasticity is very difficult to detect when $d<0.25$ and $\phi$ and/or $T$ are small. In particular, when the variance of the volatility is small $\left(\sigma_{\eta}^{2}=0.01\right)$, the power strongly depends on the value of the autoregressive parameter, $\phi$. Even if the sample size is large, the power gets reasonably close to one only for large values of $\phi$. On the other hand, increasing the variance to $\sigma_{\eta}^{2}=0.1$ increases the power, but unless the sample size is large enough, large values of $\phi$ are still required for the power to be satisfactory. When $\phi=0$, only if the sample size is very large and the long-memory parameter, $d$, is approximately greater than 0.35 , the power is reasonable. However, the dependence of the power on $d$ does not seem to be so strong as it is on the autoregressive parameter.

To summarize, we can state that the Box-Ljung test seems to have slightly higher power than the Peña-Rodriguez test for the LMSV models considered in this article. Moreover, the $Q(K)$ test has higher power against LMSV alternatives when it is implemented on squared or absolute returns than on log-squared returns. However, given that this test may have important size distortions when it is applied to the squares of Student's $t$ white noise series, we recommend the use of the absolute value transformation. In the context of short-memory ARSV models, Harvey and Streibel (1998) also conclude that the absolute transformation is more appropriate when comparing the power of a new test for heteroscedasticity with the power of the Box-Ljung test. Finally, the conclusions for the Peña-Rodriguez statistic $D(K)$ are very similar to those reported for $Q(K)$.

\section{EMPIRICAL APPLICATION TO THE IBEX-35 AND S\&P 500 INDEXES}

In this section, the previous results are illustrated with two empirical examples. Figure 7 plots, in the first row, a series of daily returns for the IBEX-35 index of the Madrid Stock Exchange observed from January 7, 1987, to December 30, 1998 (2991 observations), together with the correlograms of the log-squared, squared, and absolute observations. It also plots, in the bottom panels, a series of daily returns for the S\&P 500 index of the New York Stock Exchange (NYSE) observed from January 4, 1982, to September 23, 1994 (3218 observations), together with the correlograms of the same three transformations. The IBEX-35 returns have been filtered to remove a small autocorrelation of order one and the effect of three outliers, the minicrash on the NYSE (October 13, 1989), the kidnapping of 

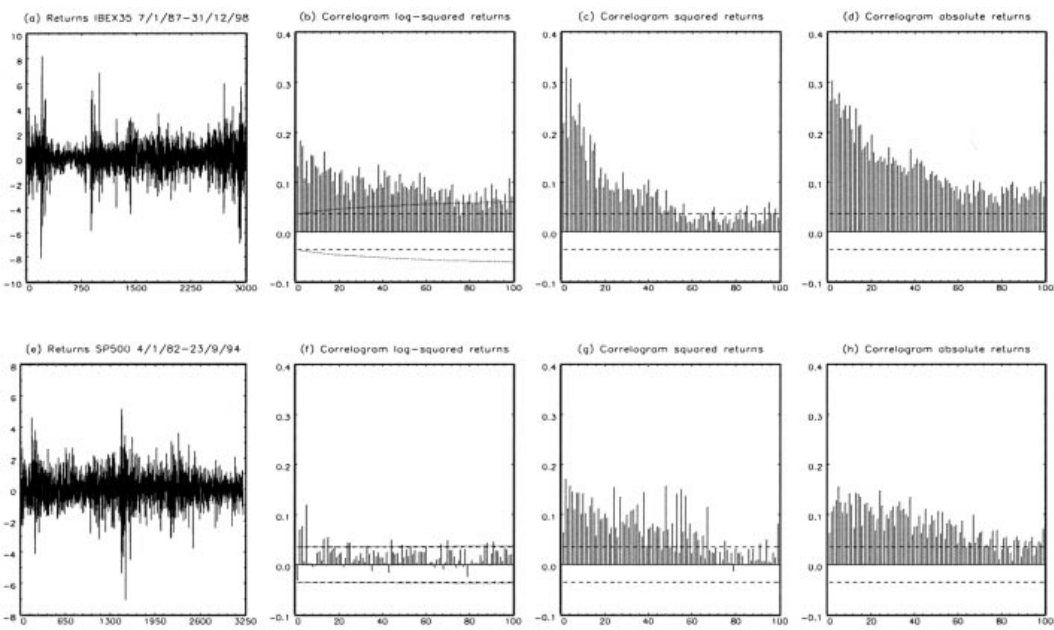

Figure 7 Daily returns of the IBEX 35 from January 7, 1987, to December 30, 1998, and the S\&P 500 from January 4, 1982, to September 23, 1994, together with the correlograms of log squared, squared, and absolute returns.

Gorbachev (August 19, 1991), and a devaluation of the Spanish peseta in the European monetary system (May 13, 1993). Similarly the S\&P 500 returns have been filtered to remove the effect of some important drops in the NYSE, namely, the minicrash on September 11, 1986, "Black Monday" (October 19, 1987), and the already mentioned minicrash of October 1989. All the correlograms are displayed with the usual 95\% Barlett confidence intervals. In the case of the log-squared returns, we also represent the $95 \%$ confidence bands obtained with the asymptotic variance given in Equation (8) replacing $\rho(k)$ by its corresponding sample counterpart, $r(k)$. Nevertheless, notice that, under the null hypothesis, $\mathrm{H}_{0}: \rho(j)=0$ for $j \geq k$, the variance of $r(k)$ in Equation (8) coincides with the classical expression in Anderson and Walker (1964) and the recursive confidence bands for $r(k)$ become the same as those obtained in linear models with i.i.d disturbances [see Brockwell and Davis (1991, pp. 223-224)].

Figure 7 shows that, in both indexes, the returns move randomly around zero while they exhibit volatility clustering. Moreover, the kurtosis of the IBEX-35 returns is 8.321 and that of the S\&P 500 returns is 6.886, indicating marginal distributions with fat tails. The evidence for conditional heteroscedasticity in the IBEX-35 returns is overwhelming. The $Q(10)$ statistics for log-squared, squared, and absolute returns are 609.28, 1729.1, and 1997.2, respectively, which are highly significant. Similar results are obtained using other lags and/or the $D(K)$ statistic. Furthermore, the sample autocorrelations of log-squared and absolute returns, displayed in Figure 7(b and d) are clearly significant even for high lags, with a 
very slow decay toward zero, suggesting long memory in volatility. However, the long-memory property is not so clear when looking at the correlogram of squared returns.

With respect to the S\&P 500 returns, the evidence for conditional heteroscedasticity is not clear in the correlogram of log-squared returns that only displays significant correlations at very few and low lags (see Figure 7(f)). However, the sample autocorrelations of squared and absolute returns are clearly significant. The $Q(50)$ statistics for these two transformations are 1810.54 and 1668.31 , respectively, which are also highly significant. Furthermore, the possible long-memory property of the volatility only arises in the absolute returns, where the correlations are small in magnitude but decay very slowly to zero (see Figure 7(h)). Therefore, in agreement with our Monte Carlo results, the presence of heteroscedasticity is clearer if squared or absolute returns are used instead of log-squared returns, while the long-memory property seems to be more evident in absolute returns than in squared returns.

\section{CONCLUSION}

In this article, we have analyzed the properties of the autocorrelations of logsquared, squared, and absolute returns generated by LMSV models, comparing them with the autocorrelations of the underlying log-volatility process. We show that these autocorrelations are typically rather small and well below the autocorrelations of the volatility, making the identification of potential conditional heteroscedasticity and long memory a difficult task. Furthermore, this problem is worsened because of the severe finite sample bias of the sample autocorrelations, especially in the more persistent cases, where it hardly vanishes even for large sample sizes. Hosking (1996) proposes functions of the sample autocorrelations that are less biased. However, whether these statistics form a suitable basis for model identification for long-memory time series has not been investigated yet. On the other hand, it is also worth pointing out that there are some popular semiparametric estimators of the parameter $d$ in LMSV models, based on the sample autocorrelations of log-squared, squared, and absolute returns, which are typically negatively biased [see, e.g., Andersen and Bollerslev (1997), Bollerslev and Wright (2000), Deo and Hurvich (2001), and Crato and Ray (2002)]. The biases found in these estimators might be related to the negative biases of the sample autocorrelations reported in this article.

As well as studying the problem of the bias in the sample autocorrelations, we have also analyzed the asymptotic distribution of the sample autocorrelations for $\log$-squared returns when $d<0.25$ and we have shown that the asymptotic variance provides an adequate approximation to the finite sample variance. However, if $d \geq 0.25$, which is a more interesting case from an empirical point of view, the asymptotic distribution of the sample autocorrelations is still unknown. Further research is required in this area.

In general, it has been shown that the sample autocorrelations of log-squared returns have the smallest standard deviations, but, in turn, their biases are severe 
relative to the magnitude of the autocorrelations. Therefore this transformation does not seem appropriate to identify the presence of conditional heteroscedasticity. Alternatively, when absolute and squared returns are considered, the standard deviations of the sample autocorrelations are smaller for the former and the relative biases are similar in both cases or slightly smaller in the absolute returns. Consequently it seems that identification of conditional heteroscedasticity is clearer using the correlogram of absolute values.

On the other hand, our simulation study on portmanteau tests for joint uncorrelatedness in LMSV models show that the Box-Ljung and Peña-Rodriguez tests have low power when either the fractional parameter is small and/or the variance $\sigma_{\eta}^{2}$ is close to zero. Furthermore, these statistics can detect spurious heteroscedasticity in the presence of homoscedastic heavy-tailed series when squared returns are used. Consequently it seems more convenient to apply the portmanteau tests to the autocorrelations of the absolute values.

Finally, it would also be interesting to study the properties of the Box-Ljung statistic as a diagnostic checking tool for conditionally heteroscedastic models with long memory. In the GARCH framework, Li and Mak (1994) prove that the autocorrelations of squared residuals are useful in checking the adequacy of nonlinear $\mathrm{ARCH}$ specifications and derive some useful diagnostic tools for such models. Bollerslev and Mikkelsen (1996) use the Box-Ljung statistic for the absolute and squared standardized residuals to check for the adequacy of several fitted GARCH-type models, including those with long memory. However, as they point out, little is known about the theoretical properties of the Box-Ljung statistic for such models. The same can be applied to the SV framework, where, as far as we know, this topic has not yet been studied.

Received April 25, 2002; revised March 25, 2003; accepted May 12, 2003

\section{REFERENCES}

Andersen, T. G., and T. Bollerslev. (1997). “Heterogeneous Information Arrivals and Return Volatility Dynamics: Uncovering the Long Run in High Frequency Returns." Journal of Finance LII, 9751005.

Andersen, T. G., T. Bollerslev, F. X. Diebold, and H. Ebens. (2001a). “The Distribution of Realized Stock Return Volatility." Journal of Financial Economics 61, 4376.

Andersen, T. G., T. Bollerslev, F. X. Diebold, and P. Labys. (2001b). "The Distribution of Realized Exchange Rate Volatility." Journal of the American Statistical Association 96, 4255.

Anderson, T. W., and A. M. Walker. (1964). "On the Asymptotic Distribution of the Autocorrelations of a Sample from a Linear Stochastic Process." Annals of Mathematical Statistics 35, 12961303.

Baillie, R. T., T. Bollerslev, and H. O. Mikkelsen. (1996). "Fractionally Integrated Autoregressive Conditional Heteroskedaticity." Journal of Econometrics 74, 330.

Baillie, R. T., and H. Chung. (2001). "Estimation of GARCH Models from the Autocorrelations of the Squares of a Process." Journal of Time Series Analysis 22, 631650. 
Bollerslev, T. (1988). “On the Correlation Structure for the Generalized Autoregressive Conditional Heteroskedastic Process." Journal of Time Series Analysis 9, 121131.

Bollerslev, T., and H. O. Mikkelsen. (1996). "Modeling and Pricing Long Memory in Stock Market Volatility." Journal of Econometrics 73, 151184.

Bollerslev, T., and H. O. Mikkelsen. (1999). "Long Term Equity Anticipation Securities and Stock Market Volatility Dynamics." Journal of Econometrics 92, 7599.

Bollerslev, T., and J. H. Wright. (2000). "Semiparametric Estimation of Long Memory Volatility Dependencies: The Role of High Frequency Data." Journal of Econometrics 98, 81106.

Bollerslev, T., and J. H. Wright. (2001). "High Frequency Data, Frequency Domain Inference, and Volatility Forecasting." Review of Economics and Statistics 83, 596602.

Breidt, F. J., and A. L. Carriquiry. (1996). "Improved Quasi Maximum Likelihood Estimation for Stochastic Volatility Models." In J. L. Lee, W. O. Johnson, and A. Zellner (eds.), Modelling and Prediction: Honouring Seymour Geisser. New York: Springer Verlag.

Breidt, F. J., and R. A. Davis. (1992). "Time Reversibility, Identifiability and Independence of Innovations for Stationary Time Series." Journal of Time Series Analysis 13, 377390.

Breidt, F. J., N. Crato, and P. J. F. de Lima. (1998). "The Detection and Estimation of Long Memory in Stochastic Volatility." Journal of Econometrics 83, 325348.

Brockwell, P. J., and R. A. Davis. (1991). Time Series: Theory and Methods, 2nd ed. New York: Springer Verlag.

Carnero, M. A., D. Peña, and E. Ruiz. (2004). "Persistence and Kurbsis in GARCH and Stochastic Volatility Models." Working paper $0108(05)$, Statistics and Econometrics Series, Universidad Carlos III de Madrid, Spain; forthcoming in Journal of Financial Econometrics.

Chung, C. F. (1994). "A Note on Calculating the Autocovariances of the Fractionally Integrated ARMA Models." Economics Letters 45, 293297.

Crato, N., and B. K. Ray. (2002). "Semi Parametric Smoothing Estimators for Long Memory Processes with Added Noise." Journal of Statistical Planning and Inference 105, 283297.

Dacorogna, M. M., U. A. Müller, R. J. Nagler, R. B. Olsen, and O. V. Pictet. (1993). "A Geographical Model for the Daily and Weekly Seasonal Volatility in the Foreign Exchange Market." Journal of International Money and Finance 12, 413438.

Davies, R. B., and D. S. Harte. (1987). "Tests for Hurst Effect." Biometrika 74, 95101.

Davis, R. A., and T. Mikosch. (2000). "The Sample Autocorrelations of Financial Time Series Models." In W. J. Fitzgerald, R. L. Smith, A. T. Walden, and P. C. Young (eds.), Nonlinear and Nonstationary Signal Processing. Cambridge: Cambridge University Press.

Deo, R. S., and C. M. Hurvich. (2001). “On the Log Periodogram Estimator of the Memory Parameter in Long Memory Stochastic Volatility." Econometric Theory 17, 686710.

Ding, Z., C. W. J. Granger, and R. F. Engle. (1993). "A Long Memory Property of Stock Market Returns and a New Model." Journal of Empirical Finance 1, 83106.

Fuller, W. A. (1996). Introduction to Statistical Time Series, 2nd ed. New York: John Wiley \& Sons. 
Ghysels, E., A. C. Harvey, and E. Renault. (1996). "Stochastic Volatility." In G. S. Maddala and C. R. Rao (eds.), Handbook of Statistics, Vol. 14. Amsterdam: North Holland.

Hannan, E. J. (1970). Multiple Time Series. New York: John Wiley \& Sons.

Harvey, A. C. (1998). "Long Memory in Stochastic Volatility." In J. Knight and S. Satchell (eds.), Forecasting Volatility in Financial Markets. Oxford: Butterworth Heinemann.

Harvey, A. C., and M. Streibel. (1998). “Testing for a Slowly Changing Level with Special Reference to Stochastic Volatility." Journal of Econometrics 87, 167189.

Harvey, A. C., E. Ruiz, and N. G. Shephard. (1994). "Multivariate Stochastic Variance Models." Review of Economic Studies 61, 247264.

He, C., and T. Terasvirta. (1999). "Properties of Moments of a Family of GARCH Processes." Journal of Econometrics 92, 173192.

Hosking, J. R. M. (1981). "Fractional Differencing." Biometrika 68, 165176.

Hosking, J. R. M. (1996). "Asymptotic Distributions of the Sample Mean, Autocovariances and Autocorrelations of Long Memory Time Series." Journal of Econometrics 73, 261284.

Li, W. K., and T. K. Mak. (1994). "On the Squared Residual Autocorrelations in Non Linear Time Series with Conditional Heteroskedasticity." Journal of Time Series Analysis 15, 627636.

Lobato, I., and N. E. Savin. (1998). "Real and Spurious Long Memory Properties of Stock Market Data." Journal of Business and Economic Statistics 16, 261283.

McLeod, A. I., and W. K. Li. (1983). "Diagnostic Checking ARMA Time Series Models Using Squared Residual Autocorrelations." Journal of Time Series Analysis 4, 269273.

Newbold, P., and C. Agiakloglou. (1993). "Bias in the Sample Autocorrelations of Fractional Noise." Biometrika 80, 698702.

Peña, D., and J. Rodriguez. (2002). "A Powerful Portmanteau Test of Lack of Fit for Time Series." Journal of the American Statistical Association 97, 601610.

Robinson, P. M. (2001). "The Memory of Stochastic Volatility Models." Journal of Econometrics 101, 195218.

Romano, J. P., and L. A. Thombs. (1996). "Inference for Autocorrelations Under Weak Assumptions." Journal of the American Statistical Association 91, 590600.

Serfling, R. J. (1980). Approximation Theorems of Mathematical Statistics. New York: John Wiley \& Sons.

Taylor, S. (1986). Modelling Financial Time Series. New York: John Wiley \& Sons.

Wright, J. H. (1999). "A New Estimator of the Fractionally Integrated Stochastic Volatility Model." Economics Letters 63, 295303. 